\title{
Review on Infectious Bovine Keratoconjunctivitis and its Economic Impacts in Cattle
}

\author{
Ahmed Seid* \\ Wolaita sodo university, school of veterinary medicine, Ethiopia
}

Submission: January 20, 2019 ; Published: February 08, 2019

*Corresponding author: Ahmed Seid, Wolaita sodo university, school of veterinary medicine, Wolaita sodo, Ethiopia

\begin{abstract}
Infectious bovine keratoconjunctivitis is one of the most common eye diseases of cattle and is of major eco-nomic importance in the world. It is a bacterial infection of the eye that causes inflammation and in severe cases temporary or permanent blindness. In cattle, the gram-negative bacterium Moraxella bovis is regarded as the main cause of the disease that affects cattle of all ages and occurs worldwide. Also, Moraxella bovoculi and a range of other bacteria, viruses, and environmental conditions seem to be involved. Moraxella bovis has several pathogenic mechanisms; however, only two, pili and the secretion of a $\beta$-hemolytic cytotoxin, have been determined to cause clinical disease. The pili allow the bacteria to attach to the dark cells of the corneal epithelium. The hemolysin is a pore-forming toxin that lyses corneal epithelial cells leading to ulceration and causes lysis of bovine leukocytes. The virulence of Moraxella bovis is influenced by both host and environmental factors. It is one of the examples of the diseases that may cause production losses in both dairy and beef farms in many countries.

The economic impact of the disease is significant due to its high contagious nature. Most cattle producers are familiar with this disease but may not know how to best treat it and minimize its spread within the herd. The cost and time used in treating infected cattle adds to the economic losses. The best strategies to prevention and control of an outbreak are maximizing the herd's immune status, minimizing the concentration of the Moraxella bacteria, and maintaining as irritant-free environment as possible. Treatment decisions are influenced by numerous factors such as effectiveness of the drug selected, cost, labor availability, withholding times, facilities, and availability of veterinary support. Vaccines are partially protective and cannot be completely relied upon to prevent the disease. Coming up with one solution is difficult because of all the contribution factors. Therefore, isolation and a swift reaction are keys in reducing the spread of the disease.
\end{abstract}

Keywords: Cytotoxin; Economic; Hemolysin; Infectious bovine keratoconjunctivitis

Abbrevations: IBK: Infectious bovine keratoconjunctivitis; BVD: Bovine Virus Diarrhea; IBR: Infectious Bovine Rhinotracheitis; BHV1: Bovine Herpes Virus 1; RTX: Repeats in-Toxin; FAT: Fluorescent Antibody Testing; PCR: Polymerase Chain Reaction

\section{Introduction}

Infectious bovine keratoconjunctivitis (IBK), or commonly known as pinkeye is a highly contagious and infectious ocular disease of cattle characterized by conjunctivitis and ulcerative keratitis, which occurs worldwide [1]. The disease also occurs in other livestock [2] and wildlife [3] and is generally regarded as a multifactorial disease. The most common causative agent of IBK is Moraxella bovis (M. bovis) [4]. The pathogenesis of the disease is influenced by many factors, such as season, mechanical irritation, host immune response, eyelid pigmentation, and concurrent presence of pathogenic bacteria, and strain of M. bovis [5]. Also, Moraxella bovoculi and a range of other bacteria, viruses, and environmental conditions seem to be involved [6].

At the present time, it is not known if Moraxella bovoculi (M. bovoculi) plays a primary or secondary role in the pathogene sis of IBK [7]. For a long period of time it had been thought the bacterium M. bovis was the primary cause of IBK. However, M. bovoculi can be isolated with or without $M$. bovis from eyes of cattle with IBK. Morever, several other infectious agents such as Adenovirus, Mycoplasma, Branhamella (Neisseria), and Listeria have been recovered from the eyes of cattle showing clinical signs like those seen in Moraxella-induced IBK [8].

There are a lot of contributing factors involved with the disease IBK. These include environmental factors like bright UV sunlight, conditions in the paddock like long stalky grass, dust and overhead hay feeders. Nutritional deficiencies also play a role with vitamin $\mathrm{A}$, and the minerals copper and selenium. A high concentration of face flies, breeds of cattle lacking eye pigment and young cattle as well as compromised immunity from other viruses such as Bovine Virus Diarrhea (BVD) [9]. 
Therefore, the objectives of this paper are

a. To give an overview on infectious bovine keratoconjunctivitis cause, occurrence, predisposing factors, method of spread and treatment and as well as its control and prevention.

b. To highlight the economic impacts of the disease (IBK) in cattle producers.

\section{Overview on Infectious Bovine keratoconjunctivitis}

Infectious bovine keratoconjunctivitis is a bacterial eye disease of cattle. The disease is perceived to be of economic importance due to poor thrift in affected animals. The financial loss is due to decreased weight gain, increased treatment costs, and market discounts due to eye disfigurement and blindness. Certain strains of $M$. bovis can produce pit-like depressions in conjunctival and corneal epithelial cells causing impaired vision in affected animals. This disease is the most common condition affecting beef and dairy heifers, and the second most common disease of nursing calves greater than three weeks old [10].

\section{Etiology}

The gram-negative rod bacterium $M$. bovis is the most primary organism incriminated to cause IBK in cattle and the most frequently isolated. The bacterium adheres to the cells via its fimbriae and pili proteins and produces $\beta$-hemolysin toxins which lyse the corneal epithelial cells [11]. Apart from the etiologic agent $M$. bovis, many factors including exposure to UV light, accumulation of dust and trauma at ocular region etc., predisposes the infection. The ability of $M$. bovis to cause the disease is influenced by host (the cattle) and environmental factors [10].

There are also several pathogens associated with IBK in cattle, such as Bovine Herpes Virus 1 (BHV 1) which is the causative agent of Infectious Bovine Rhinotracheitis (IBR). However, M. bovis has thus far been the only organism demonstrated to cause IBK in cattle [12]. There are other organisms which can result in severe conjunctivitis and edema of the cornea but they are not known to cause central corneal ulceration [13].

Moraxella bovoculi is a recently described bacterial species that associates with outbreaks of IBK [14]. This new species of Moraxella can be distinguished from two other Moraxella species, M. bovis and M. bovis, since phenylalanine deaminizes activity, as well as divergence at 6 housekeeping genes, and genetic variation within a large ribosomal RNA (rRNA) encoding locus [15]. Moraxella bovoculi has not been reported to cause IBK. However, $M$. bovoculi isolates do contain known pathogenesis factors including a Repeats in-Toxin (RTX) class operon which encodes a cytotoxin that lyses and kills neutrophils and corneal epithelial cells [16], and a pili (fimbriae) gene which is required for adherence to the corneal epithelium by $M$. bovis [17]. The extent of host range, niche specialization, and genetic diversity of $M$. bovoculi is unknown. In addition to IBK cases, $M$. bovoculi has been detected in ocular secretions from horse and reindeer conjunctivitis cases [18], IBK asymptomatic cattle [19], as well as human respiratory tracts [20] and dog teeth [21]. Other causes like M. bovis, M. catarrhalis, Neisseria ovis, and Aspergillus flavus were also isolated from IBK in cattle and other ruminants [22].

\section{Epidemiology}

Infectious bovine keratoconjunctivitis is seen worldwide but mainly in areas with high temperature climates and thus is widespread in Asia, Africa and all-American continents. It is also seen in parts of Europe and UK. In seasonal countries, this disease is most prevalent in the summer months and it usually seen in young animals. During the warmer months, fly numbers are higher and intense sunlight and dust predispose the eye to infection [23].

\section{Occurrence}

Infectious bovine keratoconjunctivitis is a highly contagious and infectious bacterial eye disease in cattle which occurs worldwide [4]. It is mainly a disease of young cattle commonly occurring in their first summer. Calves are more susceptible to infection than adults but immunologically naïve cattle can be severely affected when the herd has not been previously exposed [24,25]. Severe outbreaks may occur in older cattle if they have never been exposed to the disease. After infection, cattle develop a temporary immunity which lasts up to a year. Exposure to the causative agents in following years gives further immunity, usually without eye changes being obvious [26]. Natural outbreaks usually peak in the third or fourth week, when as much as $80 \%$ of a herd may be infected [27]. Variations, among cattle in breeds, the susceptibility to IBK have been demonstrated Hereford cattle were found to be more susceptible compared with all other purebreds such as Angus and Bos Indicus breeds [5].

Infection can occasionally persist in a few animals and these are a source of infection in the following summer. The infection rate increases to a peak about 3-4 weeks after the first cases appear, and then gradually decreases. The prevalence of IBK in districts and on individual farms varies from year to year, depending on seasons and weather, the fly population and whether cattle are grazing long grass. On some farms there may be only occasional cases while on others $60-80 \%$ of cattle may be affected in very severe outbreaks [26].

\section{Predisposing (Risk) factors}

The bacteria M. bovis reportedly causes IBK. However, numerous physical factors have been shown to influence the appearance of the ocular disease such as breed and age of the animal, UV light exposure, wind and pollen conditions, and pasture conditions. The presence of other infectious organisms in the tissues surrounding the eye, as well as concurrent upper respiratory infections, can cause the disease problem to be much more severe [28].

Like many diseases, IBK can be considered a complex of organisms and predisposing factors, which result in ocular changes that favor bacterial colonization of the eye. Predisposing factors are a largely variable component in initiation of disease and may be a more important component of the IBK ocular disease com- 
plex when dealing with less virulent strains of Moraxella. Other gram-negative bacterial cocci related to M. bovis, Moraxella ovis (formerly Branhamella ovis), and M. bovoculi have been isolated from clinical cases of IBK. A newly isolated strain of bacteria known as M. bovoculi may play an important role in IBK but research has not confirmed this. Other problems such as physical trauma or trauma due to squamous cell carcinoma may also predispose the eye to secondary bacterial infection [29].

And, Mycoplasma, Chlamydia spp., bovine herpes virus-1 and bovine adenovirus, are among the microbial agents suspected to predispose cattle to Moraxella colonization [30] or to add to the severity of IBK [31]. Mycoplasma bovis can cause eye infections resembling those seen with Moraxella bovis as well [32].

\section{Method of Spread (Transmission)}

Infectious bovine keratoconjunctivitis is transmitted by direct contact, aerosols and fomites. Flies may serve as mechanical vectors of the bacteria M. bovis [5]. The face fly Musca autumnalis is the important species in transmission of M. bovis. Moreover, the ocular and nasal discharges of infected animals can carry the pathogens, hence direct transmission from animal-to-animal contact, contaminated equipment and animal handlers can also transmit the disease [33]. Transmission occurs when a non- infected animal meets secretions infected with $M$. bovis. Secretions from the eye, nose, or vagina can be infected. Carrier animals can shed the organism for long periods of time so they are an important factor in the spread of the disease and its survival over winter. When the eyes of a carrier animal are irritated, its tear production increases and promoting the shedding of M. bovis [34]. And, eye irritation from dust, bright sunlight, thistles and long grass can cause lacrimation which attracts flies. The flies feed on the infected secretions and move from animal to animal, this spreading the bacteria within the herd of cattle [9].

\section{Pathogenic Mechanisms of M. bovis}

The pathogenic strains of $M$. bovis are piliated strains that initially bind through their pili to receptors on the surfaces of corneal epithelial cells [35]. The bacterium adheres to the cells via its fimbriae and pili proteins and produces $\beta$-haemolysin toxins which lyse the corneal epithelial cells [11]. Moraxella bovis also secretes cytotoxic toxin and pathogenic fibrinolysis, phosphatase, hyaluronidase and aminopeptidases. The bacterial membrane proteins and lipopolysaccharide are also pathogenic [36]. Moraxella bovis invades the lacrimal and tarsal glands of the eye, causing keratitis, opacity, uveitis, aqueous flare and corneal ulcers. Non-pathogenic strains of $M$. bovis exist, strains that do not produce pili or cytotoxins are much less capable of producing clinical disease [30]. And, the hemolytic and cytolytic activity from culture filtrates of $M$. bovis isolated from cattle with IBK has been reported recently and this suggests a possible role for gram-negative cocci in the pathogenesis of IBK [37].

\section{Clinical Signs}

Infectious bovine keratoconjunctivitis is ocular disease of cattle, which is clinically characterized by corneal ulceration, edema, blepharospasm, photophobia, ocular pain, lacrimation, corneal perforation and permanent blindness in severe cases $[38,39]$. Blepharospasm and photophobia suggest IBK is painful and pain mitigation therapies may be useful adjuncts to antibiotic therapy by improving animal welfare and reducing weight loss [40]. Since blepharospasm, photophobia and ocular discharge are the earliest indications of IBK, suggesting that detection occurs only once the condition is quite advanced [25]. There are four stages of IBK. The disease may resolve at any of these stages while, without treatment, the most severe cases will progress through all four stages [34].

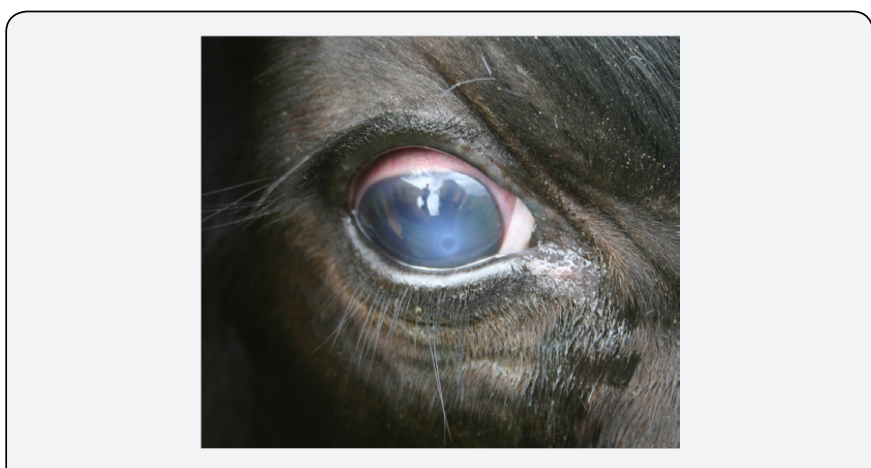

Figure 1: Watery ocular discharge and cloudy grey appearance of the cornea [34].

a. Stage I: This stage is indicated when cattle's have excessive tearing and increased sensitivity to light. They will blink frequently and there is redness along the eyelids. Cattle will often seek shade, which will decrease their grazing time. Pain associated with IBK also decreases their feed intake. Stage I will progress to a small ulcer in the center of the cornea which appears as a small white spot (Figure 1). The cornea develops a slightly cloudy grey appearance due to inflammation [34].

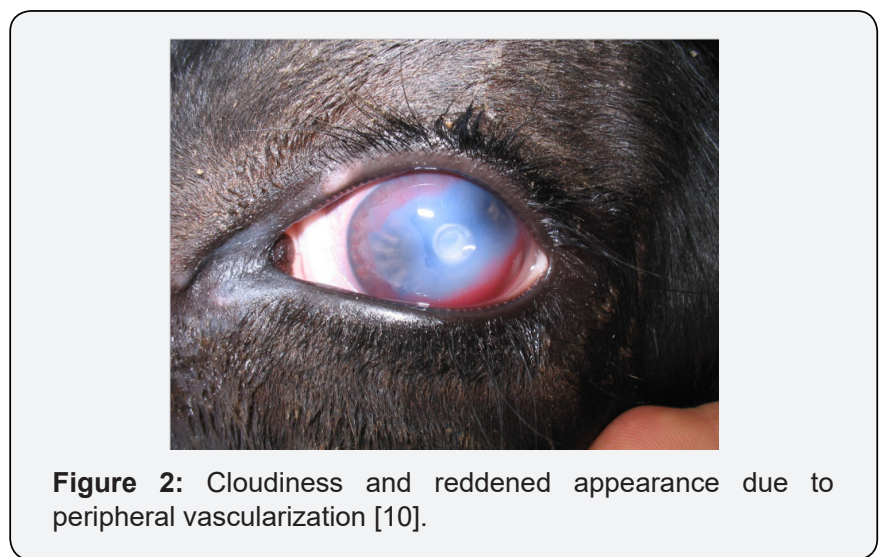

b. Stage II: The clinical signs described in Stage I continue, but this stage is indicated when the ulcer spreads across the cornea. As more inflammation occurs, the cornea becomes increasingly cloudy. At this point, some of the dark color of the iris can still be seen. Blood vessels from the outside portion of the cornea begin to grow across the cornea to help with healing (Figure 2). These blood vessels make the cornea appear pink, which is how the disease received its name [34]. 
c. Stage III: This is indicated when the ulcer covers most of the cornea and the inflammation continues to spread into the inner parts of the eye. When this occurs, the inside of the eye fills with fibrin, which is a plus-like substance that gives the eye a yellow appearance versus the typical brown appearance (Figure 3) [34]. The hemolytic M. bovis strains produce a pore forming cytotoxin [41] that promotes the development of corneal ulcers by lysis (death) of corneal epithelial cells [42].

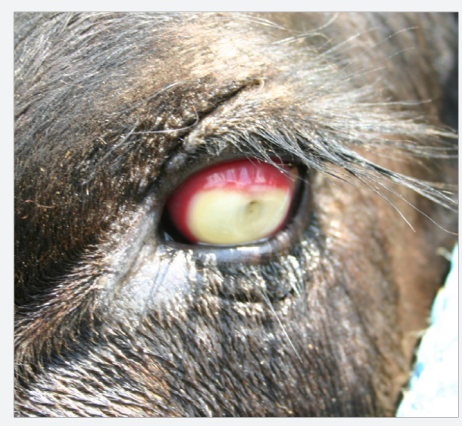

Figure 3: Yellowish appearance due to ulceration and fibrin [34]

d. Stage IV: Some animals recover spontaneously in three to five weeks, the ulcer heals and reduces, leaving a scar. In some cases, the process becomes chronic, and the opacity takes 1-2 months to resolve. In other cases, depending on the severity of the disease, a white scar may be present even after full resolution of the disease. Occasionally, perforation of the corneal ulcer results in iris prolapse, in which case, blindness may result. This stage becomes obvious when the ulcer extends completely through the cornea, and the iris may protrude through the ulcer (Figure 4). The iris will become stuck in the cornea even after healing [34].

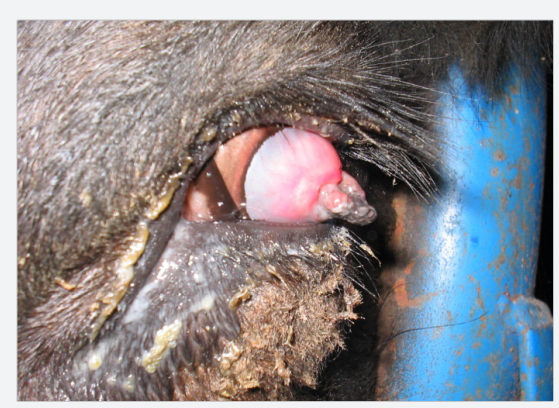

Figure 4: Perforation of the corneal ulcer results in iris prolapse [34].

\section{Diagnosis}

The clinical examination of IBK revealed mild to severe swelling surrounding affected eyes, and profuse lacrimation. Lesions typically affected either one or both of eyes, and involved the eyelid skin, conjunctiva and corneal opacity [33]. And season and history of infection and presence of flies will raise suspicion of IBK before an animal is examined. Pathology remains confined to the eye and does not reach the bloodstream [23]. On clinical examination, early disease is detectable as a raised area of cloudiness in the cornea indicating keratitis [43].

Ocular secretion specimens were collected by inserting a separate sterile swab into the inferior conjunctival fornix, and then directly inoculating the secretions on blood agar plates. And inoculated plates were subsequently streaked for isolation and incubated aerobically for $24 \mathrm{hrs}$. at $37^{\circ} \mathrm{C}$ and then examined for bacterial colonies morphologically characteristic of $M$. bovis. The colonies typical of $M$. bovis were subculture and identified, by using described morphologic and biochemical criteria [44].

The causative organism is identified based on cultural, morphological and biochemical characteristics [45]. Characteristic hemolytic colonies are observed on blood agar where it forms small, round, shiny, friable colonies but no colonies were developed on MacConkey agar plate. The pattern of hemolysis was very peculiar 1-2 mm diameter with corrosion of the agar at the edges of colony. Further, some of the colonies were found to be surface spreading. The organism is gram negative diplococci resembling tumbles, non-motile, catalase and oxidase positive. Gelatin agar is liquefied by the organism within in $24 \mathrm{hrs}$ of stab culture and able to auto agglutinate normal saline in sugar tubes [23].

Bacteriological examination revealed the production of virulent factors such as hemolysin and proteolytic enzyme production which could have caused opacity or cloudiness of the affected eye [46]. However, fimbriae also help in colonization of the organism in cornea along with capsule, the main virulence factor of $M$. bovis and the spreading nature of the hemolysis may be due to the presence of fimbriae which is also responsible for the auto agglutination of normal saline [47]. Further the laboratory results are correlated with clinical evidence such as blepharospasm, epiphora, photophobia, chemosis, corneal edema, corneal ulceration and blindness.

Fluorescent antibody testing (FAT) is available for identification and the bacterium may be visible on smears of lacrimal secretions. Polymerase chain reaction (PCR) has become an important tool for research and clinical diagnosis of infectious diseases. Multiplex real-time PCR assay was developed for the detection and differentiation of M. bovis, M. bovoculi and M. bovis [48,49].

\section{Differential Diagnosis}

Differential diagnosis includes traumatic conjunctivitis is usually easily differentiated because of the presence of foreign matter (e.g. grass awns) within conjunctival sac of the eye or evidence of a physical injury [50]. Unlike IBK, cases of bovine iritis rarely develop corneal ulceration or prulent ocular discharge, as the pathology is limited to the uveal structures. And, IBR causes conjunctivitis within rare blepharospasm and there is normally no corneal involvement [51]. Mycoplasma bovis has been isolated from the eyes of steers with an outbreak of severe conjunctivitis with corneal opacity, ulceration, and involvement of the eyelids with marked swelling was prominent. Conjunctivitis is prominent in other mycoplasmal infections that produce keratoconjunctivitis [52]. Moreover, chlamydial keratoconjunctivitis presents with identical 
clinical findings but has a protracted course despite treatment and a higher morbidity [53].

\section{Treatment}

Effective treatment of IBK can be done by use of a specific antimicrobial therapy along with proper manage approach. Early treatment of cattle with IBK is important, first for a successful outcome for the affected individual animal and then to stop the shedding of the bacteria, decreasing the risk of transmission to other cattle [54]. Appropriate antimicrobial selection requires knowledge of antimicrobial sensitivities and distribution in ocular tissues and tears. While therapeutic efficacy is affected by the frequency and mode of drug delivery, variations between intensive and extensive enterprises dictate the practical method of antimicrobial delivery. Specific recommendations for antimicrobial therapies targeting Australian IBK outbreaks are dependent upon antimicrobial pharmacokinetics, drug regulations and associated costs [55]. Generally, effective treatment of IBK is very important, as in untreated cases the corneal opacity may lead to corneal ulceration and blindness in turn it finally leads to production loss of animals. Drugs may be delivered to the eye in several ways: subconjunctival injection, topical application and systemic administration and in severe cases surgical treatment options are indicated.

Subconjunctival injection: Subconjunctival administration of antimicrobials [56] aims to reduce treatment costs and total dosages of drug while achieving higher ocular drug concentrations [57]. This probably led to some direct diffusion across the sclera and choroid; alternatively, the drug may gradually leak from the injection site, entering the tear film and eventually the eye via the cornea as if it were applied topically [58]. It also provides pharmacological advantages over deep muscle administration. Most importantly, lower dosages may be used which yield higher ocular concentrations. Difficulty of subconjunctival administration is a drawback which must be considered. Penicillin and aminoglycosides are the most commonly used subconjunctival preparations [59]. Although these drugs result in high ocular concentration, healing rates are not markedly different from deep muscle parenteral oxytetracycline [57].

Topical application: Topical administration of antimicrobial formulations has been recommended as a potentially cost-effective and less labor-intensive method for treatment of IBK [57]. Showing much promise for topical administration is oil- based formulations of benzathine cloxacillin which reduces the shedding of $M$. bovis and hasten the resolution of corneal ulcers [60]. Topical instillation of silver nitrate (1\%) and zinc sulphate $(0.4 \%)$ eye drops along with oxytetracycline parenterally, twice daily for 7-15 days to all the infected animals, which also exhibited corneal opacity were found to be more effective and led to cure within fortnight. Zinc sulphate is antiseptic, immunostimulant and astringent. It is reported that in catarrhal conditions of conjunctiva, application of zinc sulphate lotion had a proven recovery in later stage of acute infection [61]. It is also reported that zinc sulphate act as integral part of several enzymes important for wound heal- ing and ophthalmic solution is used as mild astringent for relief of eye irritation [62].

Systemic administration: Systemic antimicrobial therapy has been recommended as to target $M$. bovis located within lacrimal glands and nasal passages. Drugs administered systemically may enter the eye via the tear film or through the perilimbal or intraocular circulation. Generally, lipophilic drugs achieve higher intracorneal and intraocular concentrations and are more effective at penetrating the blood-tear barrier than hydrophilic drugs. Elimination of $M$. bovis in calves with IBK has been demonstrated following parenteral treatment with oxytetracycline [63] or florfenicol [64].

Surgical treatment options: Surgical treatment options that have been used in treating cattle with IBK include third eyelid flaps and tarsorrhaphy. In cases where globe rupture has occurred or where severe scar formation and globe protrusion represents a potential liability to the animal, exenteration may be indicated.

\section{Controls and Prevention}

Management practices that reduce the risk factors associated with IBK are the most effective tools in decreasing the incidence of the disease. Topping pastures can be a good way to reduce seed heads, and thistles which can irritate the eye. Good quality nutrition and minerals available always, will improve the overall condition of the cattle and decrease the incidence of this disease. The pre-corneal tear film is essential in eye defense mechanisms as tears wash away pathogens and tear proteins are an important part of protecting the eye. With a lower incidence rate of the disease, the overall concentration of the bacteria on the farm will be lowered, reducing the risk of a large outbreak. Shaded areas need to be provided to so cattle can get out of bright UV light when it is most intense.

Prevention of IBK is difficult because of the different types of M. bovis, its ability to change from one type to another, and the predisposing environmental conditions. Fly control is one of the most important factors. Insecticide impregnated ear tags in both ears has been shown to decrease the spread of disease. Alternatively, or additionally, insecticide sprays, pour-on, dusters, and back oilers can be used.

Vaccination can be done using bacterin such as pilli from the organism M. bovis. Cellular vaccine comprises of vaccines developed to prevent IBK include live, killed, whole cell or subunit vaccines [65]. Efforts to develop an efficacious vaccine have primarily focused upon the use of surface pili or cytolysin to stimulate host immunity; however, M. bovis possesses other virulence determinants that include proteases, fibrinolysins, phospholipases and other cell surface components such as outer membrane proteins. These potentially conserved antigens provide additional possibilities for vaccine development. Examination of appropriate antigen presentation is necessary to attain an adequate immune response. Further, the potential for antigenic diversity as well as epitope conversion requires continuous epidemiological surveillance of 
isolates recovered from outbreaks. Current work targeting conserved immunogens provides hope for efficacious vaccines that when used in tandem with proper management may control, if not prevent, IBK.

Most of these vaccines require a booster dose to be effective during the first year of use, then require a yearly booster thereafter. It is important to note that there are several different strains of M. bovis, many of which are not covered by vaccines. The disease symptoms can also be linked to another bacterium known as $M$. bovoculi, which is related to M. bovis. Incidentally, M. bovoculi is not included in any commercial IBK vaccine. Moraxella bovoculi appears to be associated with more severe IBK symptoms as well as cases that occur sporadically or outside the normal IBK season. In general, vaccination will help limit the number of outbreaks in a herd but may not eliminate the occurrence of disease. However, vaccination combined with careful management for the predisposing factors provides the best chance for preventing disease [66]

\section{The Economic Impacts of the Disease in Cattle}

Infectious bovine keratoconjunctivitis cause a significant economic loss throughout the world, due to a very painful condition affecting beef and dairy cattle worldwide. In Ethiopia, the disease causes economic losses arising from decreased weight gain in beef breeds, loss of milk production, short-term disruption of breeding programs, and treatment costs [67]. The bacterium, $M$. bovis is known to be responsible for this condition. It has been estimated that annual losses associated with only decreased weight gain from infected cattle exceeds 150 million dollars [68]. Major economic losses are the result of in appetence and poor weight gain in affected animals suffering from ocular pain and visual impairment.

Although IBK is rarely fatal, the associated impaired vision results in adverse economic impact of decreased weight gain, low calf growth rate, increased treatment costs, and market discounts due to eye disfigurement and blindness. It has been estimated that IBK costs cattle producers 150 million US\$ in the United States and 22million AUD\$ in Australia per annum as a result of in appetence and poor weight gain in affected animals suffering from ocular pain and visual impairment [69]. The largest economic loss is incurred through decreased growth as affected calves are on average 35-40 pounds lighter at weaning compared to healthy calves. Lower performance in post-weaning cattle also has also been documented with reduced average daily gain, 365th day weight, and final weight. Additionally, the drug cost for treatment, decreased market value due to corneal scarring, the loss of value of show and breeding stock, and reduced milk production from dairy animals also make this disease a significant economic consideration [29].

\section{Conclusion and Recommendations}

Infectious bovine keratoconjunctivitis (IBK) is infectious and a highly contagious eye disease of cattle, causes a great economic impact in both beef and dairy cattle farms worldwide. In cattle, the gram-negative bacterium Moraxella bovis is regarded as the main cause of the disease. This bacterium has several pathogenic mechanisms; however, only two, pili and the secretion of a $\beta$-hemolytic cytotoxin, have been determined to cause clinical disease. Environmental factors include UV light exposure, face fly populations, climate and pasture conditions and host factors include genetics, breed, age, nutrition, immune status and current infections influence the virulence of $M$. bovis. Carrier animals are asymptomatic but they shed the organism. M. bovis may be harbored in the nasal, ocular, and vaginal secretions; and it may be transmitted by direct contact, aerosol, or fomites. Cattles are the primary natural reservoir for M. bovis and there is a high nasal carrier state. The face fly, Musca autumnal is, is a primary mechanical vector for IBK and serves as an irritant. Though IBK is rarely fatal, it causes considerable economic losses to the cattle and dairy industries because of decreased weight gain, decreased milk production, devaluation because of eye disfigurement, and because of the high cost of treatment.

Based on the above conclusions, the following recommendations are forwarded:

a. Any cattle herd producer who has experienced IBK outbreak aware of the discomfort and loss of performance that can occur.

b. Early detection, segregation and treatment of infected stock.

c. Reduce the incidence of flies and subsequent spreading of bacteria with the application of pesticide self-application devices or ear tags and pour-on treatments.

d. Development of a breeding program that selects for pigmented eyelids and hair surrounding the eye.

\section{Acknowledgement}

a. First, I would like to thanks God, the almighty, most merciful, self -enough master; whom all creature of the world by having full of things; that no one is out of his control and he open the right way for all to believe on him.

b. In addition, I would like to thank all my families those are supporting me economically and morally in all my educational levels.

Finally, I have thanks to my friends and instructors of Wolaita Sodo University School of Veterinary Medicine for their valuable efforts and supports.

\section{References}

1. Holzhauer M, Visser IJ, van Maanen K (2004) Infectious bovine keratoconjunctivitis (IBK) in cows, clinical and lab review at four farms. Tijdschr Diergeneeskd 129: 526-529.

2. Motha MX, Frey J, Hansen MF, Jamaludin R, Tham KM (2003) Detection of Mycoplasma conjunctiva in sheep affected with conjunctivitis and infectious keratoconjunctivitis. NZ Vet J 51: 186-190.

3. Giacometti M, Janovsky M, Belloy L, Frey J (2002) Infectious keratoconjunctivitis of ibex, chamois and other caprinae. Rev Sci Tech 21: $335-345$. 


\section{Journal of Dairy \& Veterinary Sciences}

4. McConnell CS, Shum L, House JK (2007) Infectious bovine keratoconjunctivitis antimicrobial therapy. Aust Vet J 85: 65-69.

5. Snowder GD, van Vleck LD, Cundiff LV, Bennett GL (2005) Genetic and environmental factors associated with incidence of infectious bovine kkeratoconjunctivitis in preweaned beef calves. J Anim Sci 83: 507-518.

6. Angelos JA (2010) Moraxella bovoculi and infectious bovine keratoconjunctivitis: cause or coincidence. Vet Clin North Am Food Anim Pract 26: 73-78.

7. Angelos JA, Ball LM, Byrn BA (2011) Minimum inhibitory concentrations of selected antimicrobial agents for Moraxella bovoculi associated with bovine keratoconjunctivitis. J Vet Diagn Invest 23(3): 552-555.

8. Quinn PJ, Carter ME, Markey B, Carter GR (2002) Clinical veterinary microbiology. Mosby, Edinburgh pp. 284-286.

9. Moore A (2017) Preventing pink eye in young cattle. Kellogg rural leaders Programmed p.1-28

10. Ram K, Steve P (2011) Infectious keratoconjunctivitis (Pink eye in cattle). Animal health spotlight veterinary medicine extension p. 1-5

11. Billson FM, Harbour C, Michalski WP, Tennent JM, Egerton JR, et al. (2000) Characterization of hemolysin of Moraxella bovis using a hemolysin-neutralizing monoclonal antibody. Infect and Immun pp. 3469-3474.

12. Angelos JA, Lane VM, Ball LM, Hess JF (2010) Recombinant Moraxella bovoculi cytotoxin-ISCOM matrix adjuvanted vaccine to prevent naturally occurring infectious bovine keratoconjunctivitis. Vet Res Commun 34: 229-239.

13. Gould S, Dewell R, Tofflemire K, Whitley RD, Millman ST, et al. (2013) Randomized blinded challenge study to assess association between Moraxella bovoculi and infectious bovine keratoconjunctivitis in dairy calves. Vet Microbiol 164: 108-115.

14. Angelos JA (2015) Infectious bovine keratoconjunctivitis (pinkeye). Vet Clin N Am Food Anim Pract 31: 61-79.

15. Angelos JA, Spinks PQ, Ball LM, George LW (2007) Moraxella bovoculi $s p$. isolated from calves with infectious bovine keratoconjunctivitis. Int J Syst Evol Microbiol 57: 789-795.

16. Angelos JA, Ball LM, Hess JF (2007) Identification and characterization of complete RTX operons in Moraxella bovoculi and Moraxella ovis. Vet Microbiol 125: 73-79.

17. Calcutt MJ, Foecking MF, Martin NT, Mhlanga-Mutangadura T, Reilly TJ (2014) Draft genome sequence of Moraxella bovoculi strain 237T (ATCC BAA-1259T) isolated from a calf with infectious bovine keratoconjunctivitis. Genome Announc 2: 612-614.

18. Liu H, Yan J, Wang Y, Yan Q, Zhao L, et al. (2014) Isolation of Moraxella bovoculi from racehorses with keratoconjunctivitis. J Vet Diagn Invest 26: 585-587.

19. Schnee C, Heller M, Schubert E, Sachse K (2015) Point prevalence of infection with Mycoplasma bovoculi and Moraxella spp. in cattle at different stages of infectious bovine keratoconjunctivitis. Vet J 203: 92 96.

20. Nakamura S, Yang CS, Sakon N, Ueda M, Tougan T, et al. (2009) Direct metagenomic detection of viral pathogens in nasal and fecal specimens using an unbiased high-throughput sequencing approach. Plos One, 4: 421

21. Riggio MP, Lennon A, Taylor DJ, Bennett D (2011) Molecular identification of bacteria associated with canine periodontal disease. Vet Microbiol 150: 394-400.

22. Libardoni F, Charles FC, Scherer LF, Andréia V, Claudia B, et al. (2012) Moraxella bovoculi in cases of infectious bovine keratoconjunctivitis in Rio Grande do Sul, Brazil. Pesq Vet Bras 32(8): 743-746.
23. Haskell S (2008) Blackwell's five-minute veterinary consult Ruminant. Wiley-Blackwell, Oxford Merck Veterinary Manual, Infectious Keratoconjunctivitis, UK.

24. Kizilkaya K, Tait RG, Garrick DJ, Fernando RL, Reecy JM (2013) Genomewide association study of infectious bovine keratoconjunctivitis in Angus cattle. BMC genetics 14: 23.

25. Postma GC, Carfagnini JC, Minatel L (2008) Moraxella bovis pathogenicity: an update. Comp. Immunol. Microbiol Infect Dis 31(6): 449-458.

26. Walker B (2007) Pinkeye in cattle. Prime fact 336. (Replaces AGFACT A0.9.31) NSW Department of primary industries.

27. Kirkpatrick JG, Lalman D (2000) Pinkeye in cattle. Infectious bovine keratoconjunctivitis (IBK). Oklahoma cooperative extension service.

28. Stokka GL, Davidson HJ, Van Boening J (2000) (Pinkeye) Infectious bovine keratoconjunctivitis. Kansas state university cooperative extension service. p. 1-4.

29. Arnold M, Lehmkuhler J (2012) Infectious bovine keratoconjunctivitis (pinkeye) in cattle.

30. Brown MH, Brightman AH, Fenwick BW, Rider MA (1998) Infectious bovine keratoconjunctivitis: a review. J Vet Intern Med 12 (4): 259-266.

31. Scharko P (2004) Disease headaches in Kentucky: Pinkeye, anaplasmosis, and listeria. Presented at the Kentucky ruminant nutrition workshop.

32. Alberti A, Addis MF, Chessa B, Cubeddu T, Profiti M, et al. (2006) Molecular and antigenic characterization of a Mycoplasma bovis strain causing an outbreak of infectious keratoconjunctivitis. J Vet Diagn Invest 18 (1): 41-51.

33. Jeyabal L, Debdatta Ray D, Sureshkannan S, Nagarajan K, Visnuvinayagam S, et al. (2013) First report of morexella bovis infection in Indian cattle. Adv Anim Vet Sci 1(6): 202-204.

34. Whittier WD, Currin J, Currin N (2009) Pinkeye in beef cattle, College of Agriculture and Life Sciences, Virginia polytechnic institute and state university. p. 1-5.

35. Conceicao FR, Dellagostin OA, Paolichi F, Leturia AC, Gil Turnes C (2004) Molecular diversity of Moraxella bovis isolated from Brazil, Argentina and Uruguay over a period of three decades. Vet J 167: 53-58.

36. Prieto CI, Aguilar OM, Yantorno OM (1999) Analyses of lipopolysaccharides, outer membrane proteins and DNA fingerprints reveal intraspecies diversity in Moraxella bovis isolated in Argentina. Vet Microbiol 70(3/4): 213-223.

37. Cerny HE, Rogers DG, Gray JT, Smith DR, Hinkley S (2006) Effects of Moraxella (Branhamella) ovis culture filtrates on bovine erythrocytes, peripheral mononuclear cells, and corneal epithelial cells. J Clin Microbiol 44: 772-776.

38. Abdullah FFJ, Adamu L, Osman AY, Haron AW, Saharee AA (2013) Clinical management of stage III infectious bovine keratoconjunctivitis associated with Staphylococcus aureus in a dairy cow: a case report. J Agricul Vet Scien 4: 69-73.

39. Alexander D (2010) Infectious bovine keratoconjunctivitis: a review of cases in clinical practice. Vet Clin North Am Food Anim Pract 26: 487503.

40. Williams DL (2010) Welfare issues in farm animal ophthalmology. Vet Clin North Akm. Food Anim Pract 26(3): 427-435.

41. Clinkenbeard KD, Thiessen AE (1991) Mechanism of action of Moraxella bovis hemolysin. Infect Immun 59: 1148-1152.

42. Beard MK, Moore LJ (1994) Reproduction of bovine keratoconjunctivitis with a purified haemolytic and cytotoxic fraction of Moraxella bovis. Vet Microbiol 42: 15-33. 
43. Bedford PGC (1992) Ocular diseases. Bovine medicine: diseases and husbandry, Wiley-Blackwell, Oxford, UK, 27: 712-721.

44. Conceicao FR, Paolicchi F, Cobo AL, Gil Turnes C (2003) Antigenic relationships of Moraxella bovis isolates recovered from outbreaks of infectious bovine keratoconjunctivitis in Argentina, Brazil, and Uruguay between 1983 and 2000. Can J Vet Res 67: 315-318.

45. Collee JG, Grulo JG, Fraser AG, Marmion BP (1989) In: Mackie and McCartney. Practical medical microbiology. $\left(13^{\text {th }}\right.$ edn $)$, Churchill and living stone, New York, USA, p. 76.

46. Frank-Sandars K, Gerber JD (1981) Hydrolytic enzymes of Moraxella bovis. J Clin Microbiol 13: 269-271.

47. Hoien-Dalen PS, Rosenbusch RF, Roth JA (1990) Comparative characterization of the leukocidic and hemolytic activity of Moraxella bovis. Am J Vet Res 51: 191-196.

48. O'Connor A, Shen H, Wang C, Opriessnig T (2012) Descriptive epidemiology of Moraxella bovis, Moraxella bovoculi and Moraxella ovis in beef calves with naturally occurring infectious bovine keratoconjunctivitis (Pinkeye). Vet Microbiol 155: 374-380.

49. Shen H, Gould S, Kinyon J, Opriessnig T, O'Connor A (2011) Development and evaluation of a multiplex real-time PCR assay for the detection and differentiation of Moraxella bovis, Moraxella bovoculi and Moraxella bovis in pure culture isolates and lacrimal swabs collected from conventionally raised cattle. J appl microbiol111: 1037-1043.

50. Radostits OM, Gay CC, Hinchcliff KW, Constable PD (2006) Veterinary medicine - A textbook of the diseases of cattle, horses, sheep, pigs and goats, $\left(10^{\text {th }} \mathrm{edn}\right)$, Saunders Elsevier, Edinburgh, London, New York USA, pp. 994-996.

51. Scott PR, Penney CD, Macrae AI (2011) Cattle medicine. Division of veterinary clinical sciences Royal (Dick) school of veterinary studies university of Edinburgh Easter Bush Veterinary Centre Roslin Midlothian, UK, pp. 211-212.

52. Naglic T, Sankovic F, Madic J (1996) Mycoplasmas associated with bovine conjunctivitis and keratoconjunctivitis. Acta Vet Hung 44: 21 24.

53. Otter A, Twomey DF, Rowe NS, Pearce SG, Ker CL, et al. (2003) Suspected chlamydial keratoconjunctivitis in British cattle. Vet Rec 152: 787-788.

54. Irsik M (2009) Pinkeye in beef cattle. University of Floria College of veterinary medicine. p. 1-3.

55. McConnell CS, House, JK (2005) Infectious bovine keratoconjunctivitis vaccine development. Aust Vet J 83(8): 506-510.

56. Kibar M, Gumussoy KS, Ozturuk A (2006) Evaluation of various antibiotic treatments in calves with infectious bovine keratoconjunctivitis. Turk J Vet Anim Sci 30: 553-559.
57. George LW (1990) Antibiotic treatment of infectious bovine keratoconjunctivitis. Cornell vet 80: 229-235.

58. Slatter DH, Edwards ME, Hawkins CD (1982) A national survey of the clinical features, treatment and importance of infectious bovine keratoconjunctivitis. Aust Vet J 59(3): 69-72.

59. Abeynayake P, Cooper BS (1989) The concentration of penicillin in bovine conjunctival bacterial fluid as it pertains to the treatment of Moraxella bovis infection. J Vet Pharm 12: 25-30.

60. Diagneault J, George LW (1990) Topical benzathine cloxacillin for the treatment of experimentally induced infectious bovine keratoconjunctivitis. Am J vet Res 51: 1153-1156.

61. Hoare EW, Greig RJ (2004) Veterinary material, medical and therapeutics, Asiatic publishing house, Delhi, India, p. 179-192.

62. Tatro S (2003) A- Z drug facts, facts and comparisons, A Wolters Kluwer Company, Missouri, ( $4^{\text {th }}$ edn), pp. 1332.

63. Starke A, Eule C, Meyer H (2007) Efficacy of intrapalpebral and intramuscular application of oxytetracycline in a natural outbreak of infectious bovine kertoconjunctivitis (IBK) in calves. Dtsch Tierarztl. Wochenschr 114: 219-224.

64. Dueger EL, Angelos JA, Cosgrove S (1999) Efficacy of florfenicol in the treatment of experimentally induced infectious bovine keratoconjunctivitis. Am J of Vet Res 60(8): 960-964.

65. Funk L, O’Connor AM, Maroney M, Engelken T, Cooper VL, et al. (2009) A randomized and blinded field trial to assess the efficacy of an autogenous vaccine to prevent naturally occurring infectious bovine keratoconjunctivis (IBK) in beef calves. Vaccine 27: 4585-4590.

66. Ward H, Powell J (2017) Livestock health series pinkeye, university of Arkansas, United States department of agriculture, and County governments cooperating. p. 1-2.

67. Takele G, Zerihun A (2000) Epidemiology of infectious bovine keratoconjunctivitis in cattle in south-east Ethiopia. J Vet Med a Physiol Pathol Clin Med 47: 169-173.

68. Lane VM, George LW, Cleaver DM (2006) Efficacy of tulathromycin for treatment of cattle with acute ocular Moraxella bovis infections. J Am Vet Med Assoc 229: 557-561.

69. Hansen R (2001) New tools in the battle against pinkeye. In: In Nevada Livestock Production Annual update: Univ. of Nevada-Reno, Nevada, USA, p. 5-8.
This work is licensed under Creative

Commons Attribution 4.0 License

DOI: 10.19080/JDVS.2019.09.555774 